\title{
Alcohol consumption and its relationship to diabetes mellitus: friend or foe?
}

\begin{abstract}
The diabetes mellitus rates are increasing worldwide reaching epidemic levels. Alcohol consumption is also increasing globally. It is known that excessive alcohol consumption is an important lifestyle risk factor for several chronic conditions, including diabetes mellitus and its complications. The relationship between diabetes mellitus and alcohol is not completely elucidated. Most studies suggest a "U" shape relation of alcohol drinking and diabetes mellitus risk and its complications. The ingestion of moderate amounts of alcohol might not just decrease diabetes mellitus development risk, but also be associated to better metabolic control, decrease in some microvascular complications (retinopathy and nephropathy), decrease in macrovascular events and mortality. The benefits may be restricted not just to the moderate amount of alcohol intake, but also vary according to age gender, body mass index, ethnics and type of alcohol drinks.
\end{abstract}

Keywords: diabetes mellitus, alcohol consumption, diabetes onset, microvascular complications, macrovascular complications
Volume 6 Issue I - 2018

\author{
Alexei Volaco,' Carolina Rodrigues Ercolano² \\ 'Pontificia Universidade Catolica do Parana, Adjunct Professor in \\ Endocrinology, School of Medicine, Brazil \\ ${ }^{2}$ Pontificia Universidade Catolica do Parana, Graduate, School of \\ Medicine, Brazil
}

\author{
Correspondence: Alexei Volaco, Adjunct Professor in \\ Endocrinology, School of Medicine. Pontificia Universidade \\ Catolica do Parana, R, Imaculada Conceicao, I I55-Prado Velho, \\ Curitiba-PR, 802I5-90I, Brazil, Tel 55(4I)327I-I555, \\ Emailavolaco@yahoo.com.br, alexei.volaco@pucpr.br
}

Received: December 17, 2018 | Published: January 24, 2018
Abbreviations: DM, diabetes mellitus; T2DM, type 2 diabetes mellitus; IDF, international diabetes federation; WHO, world health organization; HbA1c, glycated hemoglobin; NIAAA, national institute on alcohol abuse and alcoholism

\section{Introduction}

The world population with diabetes mellitus (DM) is estimated at 425 million, which represents $8.8 \%$ of adults aged 20 to 79 years, and could reach 629 million by $2045 .^{1}$ Of these, 438.2 million will be of productive age. There is a slight predominance among men. In older population (65-79years), the predominance between the sexes is similar. About $90 \%$ of the cases are type 2 diabetes mellitus (T2DM). According to the International Diabetes Federation (IDF) 727 billion dollars per year are spent on diabetes care. ${ }^{1}$ The countries with the greatest amounts of individuals with DM, as expected, are the most populous, such as China, India, the United States, Brazil, and Mexico. The population with the highest prevalence $(13.7 \%)$ of DM is the Eastern Mediterranean region. In the last decades, developing countries have increased prevalence of DM. ${ }^{1,2}$

It was estimated in 2010 that $40.6 \%$ of the world's adult population consumed alcohol. ${ }^{3}$ Worldwide consumption of pure alcohol per capita in individuals older than 15years, according to World Health Organization (WHO), is on average 6.2liters per year or 13.5 grams per day. ${ }^{4}$ In 2015, in the United States, $86.4 \%$ of the population over 18years old reported they have already consumed alcohol. ${ }^{5}$ The highest consumption rates occur in developed countries, although there is great variation in consumption among the regions due to social and cultural factors. Therefore, the Central and Eastern regions of Europe concentrate the highest alcohol intake.,

In the world, in 2010, the prevalence of alcohol abuse was estimated at $11.7 \%$ in the population aged 15 to 19 years, compared to $7.5 \%$ in the total population aged 15 years or older. On the other hand, $48 \%$ of the world population has never consumed alcohol. ${ }^{4}$ The abstinent population was more prevalent in North Africa, the Middle East and South Asia and reaches $90 \%$ in Southeast Asia. ${ }^{3}$

There are more consumers of alcoholic drinks, with a lowrisk consumption pattern, among the population with a higher socioeconomic level, whereas abstinent ones are more common in the poorer social groups. However, people from lower socioeconomic status appear to be more vulnerable to the deleterious effects of alcohol consumption. Many factors may explain this situation, including the lack of resources in the treatment of comorbidities, and less access to quality health services. ${ }^{4}$ The most widely consumed type of alcoholic beverage in the world is distilled, representing $50 \%$ of consumption and is more common in the Southeast Asia and Western Pacific regions. Secondly, beer accounts for $34.8 \%$ of that consumption and predominates in the Americas region, while wine accounts for $8 \%$ of global consumption and is more consumed in Europe. ${ }^{4}$

One serving of alcoholic beverage equals $300 \mathrm{ml}$ of beer, $100 \mathrm{ml}$ of wine or $30 \mathrm{ml}$ of distilled drinks corresponding to 10 to $12 \mathrm{~g}$ of pure alcohol. ${ }^{4}$ According to the Centers for Disease Control and Prevention, alcohol consumption is defined as binge drinking in four or more doses for women, and five or more doses for men, at the same time within the last 30 days. Heavy drinking is defined by consuming 8 or more alcoholic beverages for women, and 15 or more for men per week. ${ }^{6}$ Low-risk (moderate) consumption can be estimated for 4 doses, or less, on a single day and no more than 14 doses per week for men. For women, this consumption cannot exceed 3 doses in a single day, and no more than 7 doses per week. ${ }^{4,7}$ The pattern and amount of female consumption differs from males, and this is due to both physiological and cultural differences. The woman's body is more vulnerable to the effects of alcohol due to less metabolizing capacity, lower proportion of water in the body and generally, lower weight compared to men. ${ }^{4,8}$ Children, adolescents and the elderly are typically more vulnerable to alcohol-related harms. The most common pattern of consumption among young people is binge drinking. The increase in age is associated with decreased binge drinking and increased heavy drinking. While young people tend to behave recklessly, engaging in risky accidents, elderly who drink alcohol tend to suffer unintentional accidents, as falls. ${ }^{4,9}$

\section{Relationship between alcohol consumption and the onset of diabetes mellitus}

Different studies have shown a generally beneficial relationship between alcohol consumption and the onset of T2DM. Variations may 
occur depending on the amount and pattern of consumption, type of drink, race, body mass index, age, and gender. Recent studies present results with reduced risk in the development of DM in moderate alcohol users compared with nondrinkers. ${ }^{10-13}$ Risk reduction of up to $30 \%$ has already been found in adults. ${ }^{11}$

Researchers have described a "U-shaped" relation between alcohol consumption and DM risk. Compared to the minimal or non-consumption, the risk of T2DM is lower for moderate alcohol consumption, while high alcohol consumption leads to an increased risk similar to that of nondrinkers. A meta-analysis found significant evidence, among cohort studies from different countries, that the consumption of less than 30 grams of alcohol per day is associated with the lower risk of T2DM in the general population. ${ }^{14}$ Knott et al., ${ }^{13}$ showed maximal risk reduction of $18 \%$ with consumption between 10 and $14 \mathrm{~g}$ per day. ${ }^{13}$ The protective effect of alcohol consumption on the onset of diabetes occurred in both men and women. ${ }^{11,12,15-17}$ The highest protective effect was associated with an approximate consumption of $22 \mathrm{~g}$ per day for men and $24 \mathrm{~g}$ per day for women. Above $50 \mathrm{~g}$ for women and $60 \mathrm{~g}$ per day for men, it has been described as harmful. ${ }^{11}$

The consumption pattern can also define the risk. It is suggested that episodic binge drinking is associated with increased risk of cardiovascular disease and complications when compared to individuals with frequent consumption patterns of the same amount spread over 3 to 4 days per week. ${ }^{11,13}$ It has been suggested in New Zealand adult workers, an inverse relationship between alcohol consumption and the incidence of T2DM in individuals with normal weight and overweight. The study found no protection with obesity. ${ }^{17}$ A Japanese systematic review concluded that in Japanese men with low body mass index (defined as $\leq 22.0 \mathrm{~kg} / \mathrm{m}^{2}$ ) alcohol intake increase risk of developing DM. ${ }^{18}$

In elderly (65years of age or more) moderate alcohol users has led to $40 \%$ decrease in the risk of T2DM. The Cardiovascular Health Study indicated that mild to moderate alcohol consumption was associated with a lower incidence of T2DM among the elderly. ${ }^{19}$ Knott et al., ${ }^{13}$ suggested that the lower risk of developing T2DM may be restricted to women and non-Asian populations.

Regarding the type of alcoholic beverage wine, beer and distilled drinks showed the same "U" pattern in relation to T2DM risk, although they presented different risk proportions. A recent systematic review published in 2017 concluded that the consumption of up to $80 \mathrm{~g}$ per day of wine reduces the risk of T2DM, with best rates (up to $20 \%$ reduction in the risk) if between 20 and $30 \mathrm{~g}$ per day. As for beer consumption, the same daily amount showed a $9 \%$ risk reduction and for distillates, a $5 \%$ decrease in the risk with consumption between 7 and 15 grams per day. However, the risk of T2DM increased when daily beer consumption was greater than $80 \mathrm{~g} .{ }^{16}$ Other studies have also shown beneficial effects of moderate wine consumption, especially in women. ${ }^{10,12}$ Moderate alcohol consumption may increase insulin sensitivity in women and reduce glycemia and glycated hemoglobin (HbAlc) levels in non-diabetics. ${ }^{17}$ This risk reduction is present regardless of association with healthy lifestyle habits. ${ }^{18}$

\section{Alcohol consumption in individuals with diabetes mellitus}

In a multicenter study, with 11.140 diabetic patients evaluated, $30 \%$ reported detailed alcohol consumption as 5 servings of wine and 7 servings of beer or distillate on average per week. Of these,
$4 \%$ reported extremely high intakes of more than 21 doses for men and 14 doses for women per week. ${ }^{20}$ Similar prevalence of alcohol consumption was estimated at $30.9 \%$ among elderly with at least one chronic illness such as diabetes in the United States. ${ }^{21}$

The American Diabetes Association recommends that diabetic patients limit alcohol intake to one daily dose for women and two for men. One serving corresponds to $340 \mathrm{~g}$ of beer, $140 \mathrm{~g}$ of wine or $42 \mathrm{~g}$ of distillate. The risk of hypoglycemia increases in diabetic patients who are insulin dependent or on secretagogues when they consume alcohol. ${ }^{22}$ The IDF recommends for a healthy lifestyle that the general population limit alcohol consumption for up to 2 daily doses; for diabetics, excessive alcohol consumption should be avoided for better glycemic control. ${ }^{1}$

The alcohol consumption by diabetic patients makes it difficult to adhere to the treatment, mainly to lifestyle changes. Besides preventing behavioral measures, excessive alcohol consumption impairs glycemic control. The study suggested a measure known as "brief intervention", which consists of counseling sessions by health professionals directed at diabetic outpatients who reported risk pattern on alcohol consumption, as defined by NIAAA (National Institute on Alcohol Abuse and Alcoholism). This intervention resulted in a decrease in the percentage of patients with high alcohol consumption and the study suggests that this change improved the adherence to DM treatment. ${ }^{23}$

\section{Effects of alcohol consumption on glycemic control and chronic complications of DM}

Moderate alcohol consumption may increase insulin sensitivity in women and reduce glycemia and $\mathrm{HbA} 1 \mathrm{c}$ levels even in non-diabetics. ${ }^{24}$ This risk reduction is present regardless of healthy lifestyle habits. ${ }^{25}$ There is evidence that alcohol in moderate doses improves glycemic control and progression of complications. ${ }^{26}$

It has been shown an inverse relationship between alcohol consumption and $\mathrm{HbAlc}$ values in an analysis of 38.564 diabetic adults. Compared to abstinent, consumption of 2 to 2.9 doses of alcohol per day represented a significant reduction of $0.5 \%$ in $\mathrm{HbA} 1 \mathrm{c}$ values. Worse glycemic control was identified with consumption of 3 or more doses per day and in non-alcohol drinkers. ${ }^{26}$

Regarding the development of microvascular complications, the consumption of alcohol by individuals with DM does not increase the risk of developing diabetic retinopathy, actually a meta-analysis with 15 studies identified that wine consumption may reduce the risk of this complication. ${ }^{27}$ Moderate alcohol consumption, but not heavy, had a significant reduction in the development of chronic kidney disease in individuals with DM. ${ }^{28}$ It is speculated that moderated alcohol consumption might be protective for renal vasculature and excessive alcohol consumption increases blood pressure and consequently damages the kidney. ${ }^{29}$

In individuals with DM alcohol consumption was negatively associated with peripheral neuropathy. The prevalence and rate of development of symptomatic peripheral neuropathy was higher in diabetic men who consumed alcohol daily compared to nondrinkers, possibly due to their own and additives effects of alcohol in the development of neuropathies. ${ }^{30,31}$ About macrovascular diseases, individuals with DM that consume moderate alcohol amounts are less likely to have cardiovascular events than nondrinkers. ${ }^{32} \mathrm{We}$ also found a significant reduction in mortality rates due to coronary heart disease in diabetics with daily alcohol consumption of $14 \mathrm{~g}$ or more compared 
to nondrinkers. ${ }^{33}$ In another diabetic population, with a prevalence of $30 \%$ of alcohol consumption, a reduction in cardiovascular events has also been demonstrated. ${ }^{20}$

In individuals with T2DM, consuming mainly wine reduces the risk of cardiovascular events and is related to lower mortality rates compared to non-alcohol drinkers. A reduction in the mortality of these diabetic patients who consumed mainly beer or distillates was also observed, although, no reduction in cardiovascular events has been shown. ${ }^{20}$

A $17 \%$ reduction in cardiovascular events and $13 \%$ of the causes of mortality was reported in a study that evaluated the protective effect of moderate alcohol consumption in diabetic patients. ${ }^{20}$ In agreement with these findings, a Swiss study concluded that alcohol consumption between 16 and $30 \mathrm{~g}$ per day is associated with mortality reduction by cardiovascular disease, but consumption above $30 \mathrm{~g}$ per day increased mortality. ${ }^{34}$

Abusive consumption of alcohol, otherwise, is related to an increased risk of cirrhosis which results in the development of DM (35). Alcohol abuse causes a pro-inflammatory stimulus in the liver and metabolic system that causes a systemic subclinical inflammatory state and contributes to atherosclerosis. ${ }^{36}$ Abusive alcohol consumption is associated with increased risk of DM and higher rates of diabetic complications, so this pattern of consumption should be strongly discouraged. ${ }^{11}$ According to the WHO, abusive consumption of alcoholic beverages is considered a risk factor not just for DM, but for several chronic diseases. ${ }^{4}$

\section{Pathophysiological beneficial mechanisms of alcohol consumption (vascular effects)}

Some hypotheses suggest the influence of moderate alcohol consumption on the reduction of oxidative damage, both in circulating lipoproteins and in the coronary endothelial layer, reducing the severity of coronary artery disease. ${ }^{36}$ The polyphenols present in the wine were associated with reduced DM risk. ${ }^{16}$ Ingestion of 25 to $30 \mathrm{~g}$ per day of alcohol is related to increase in adiponectin gene expression and its serum concentration. This protein is inversely associated with inflammatory activity, incidence of T2DM and positively related to increased insulin sensitivity in women. ${ }^{25}$ Significant reduction in the levels of inflammatory markers, such as tumor necrosis factor and fibrinogen, as well as improved endothelial function were positively related to moderate alcohol intake $(<25 \mathrm{~g}$ per day) in diabetic men. It has also been shown the "U" pattern of alcohol consumption and concentration of inflammatory markers, especially C-reactive protein. ${ }^{15}$ These correlations might explain the protective characteristic of alcohol to cardiovascular events in this population. ${ }^{15,37}$

\section{Conclusion}

In conclusion, DM is a prevalent chronic disease responsible for impaired quality of life and associated with early mortality. Moderate alcohol consumption reduces the risk of developing T2DM, it is potentially beneficial in metabolic control, in preventing the progression of some complications and cardiovascular events and mortality. A possible and important confounding factor is related to the consumption of alcohol and higher socioeconomic levels, which is an important protective factor for any chronic non-communicable diseases, such as DM. On the other hand, excessive consumption may increase the risk of DM, other chronic diseases and diabetic neuropathy. Excessive alcohol consumption also increases mortality from external causes such as accidents, leading to loss of life-years, especially in young men. More prospective studies are needed to access the protective and harm effects of alcohol consumption in diabetes risk, its influence on diabetic complications and the mechanisms for that. The possible protective effect of alcohol consumption also needs to be balanced with the potential risks.

\section{Acknowledgment}

None.

\section{Conflicts of interest}

None.

\section{References}

1. International Diabetes Federation. IDF Diabetes Atlas. 2017.

2. World Health Organization. Global Report on Diabetes. 2016; Isbn 978:88.

3. Shield KD, Rylett M, Gmel G, et al. Global alcohol exposure estimates by country, territory and region for 2005-a contribution to the Comparative Risk Assessment for the 2010 Global Burden of Disease Study. Addiction. 2010;108(5):912-922.

4. OMS. Global status report on alcohol and health 2014. Glob status Rep alcohol, 2014; pp. 1-392.

5. National Institute on alcohol Abuse and Alcoholism. Alcohol Facts and Statistic. 2017

6. (CDC) $\mathrm{C}$ for DC and P. Fact Sheets-Preventing Excessive Alcohol UseAlcohol. 2016

7. Drink-equivalents A. Appendix 9. Alcohol 5-9. 2017.

8. Jandrot Louka F, Louka JM. Women and alcohol. Inf Psychiatr 2015;9:1005-1025

9. National Institute on alcohol abuse and alcoholism. Older Adults. 2017.

10. Beulens JWJ, Van der Schouw YT, Bergmann MM, et al. Alcohol consumption and risk of type 2 diabetes in European men and women:Influence of beverage type and body size. The EPIC-InterAct study. J Intern Med. 2012;272(4):358-370.

11. Baliunas DO, Taylor BJ, Irving H, et al. Alcohol as a risk factor for type 2 diabetes:A systematic review and meta-analysis. Diabetes Care. 2009;32(11):2123-2132.

12. Holst C, Becker U, Jørgensen ME, et al. Alcohol drinking patterns and risk of diabetes:a cohort study of 70,551 men and women from the general Danish population. Diabetologia. 2017;60(10):1941-1950.

13. Knott C, Bell S, Britton A. Alcohol consumption and the risk of type 2 diabetes:A systematic review and Dose-Response Meta-analysis of more than 1.9 million individuals from 38 observational studies. Diabetes Care. 2015;38(9):1804-1812.

14. Li X, Yu F, Zhou Y, et al. Association between alcohol consumption and the risk of incident type 2 diabetes :a systematic review and doseresponse meta-analysis 1. Am J Clin Nutr. 2016;103(3):818-829.

15. Imhof A, Froehlich $\mathrm{M}$, Brenner $\mathrm{H}$, et al. Effect of alcohol consumption on systemic markers of inflammation. Lancet. 2001;357(9258):763-767.

16. Huang J, Wang X, Zhang Y. Specific types of alcoholic beverage consumption and risk of type 2 diabetes:A systematic review and metaanalysis. J Diabetes Investig. 2017;8(1):56-68.

17. Metcalf PA, Scragg RKR, Jackson R. Light to Moderate Alcohol Consumption is Protective for Type 2 Diabetes Mellitus in Normal Weight and Overweight Individuals but Not the Obese. $J$ Obes. 2014;2014(2014):8 
18. Seike N, Noda M, Kadowaki T. Alcohol consumption and risk of type 2 diabetes mellitus in Japanese: A systematic review. Asia Pac J Clin Nutr . 2008;17(4):545-551.

19. Djousse L, Biggs ML, Mukamal KJ, et al. Alcohol Consumption and Type 2 Diabetes Among Older Adults:The Cardiovascular Health Study. Obesity (Silver Spring). 2007;15(7):1758-1765.

20. Blomster JI, Zoungas S, Chalmers J, et al. The relationship between alcohol consumption and vascular complications and mortality in individuals with type 2 diabetes. Diabetes Care. 2014;37(5):1353-1359.

21. Ryan M, Merrick EL, Hodgkin D, et al. Drinking patterns of older adults with chronic medical conditions. J Gen Intern Med. 2013;28(10):13261332.

22. Association AD. Standards of Medical Care in Diabetes-2018. J Med Educ. 2018;41(1).

23. Engler PA, Ramsey SE, Smith RJ. Alcohol use of diabetes patients:The need for assessment and intervention. Acta Diabetol. 2013;50(2):93-99.

24. Schrieks IC, Heil ALJ, Hendriks HFJ, et al. The Effect of alcohol consumption on insulin sensitivity and glycemic status:A systematic review and meta-analysis of intervention studies. Diabetes Care. 2015;38(4):723-732.

25. Joosten MM, Grobbee DE, Van Der A DL, et al. Combined effect of alcohol consumption and lifestyle behaviors on risk of type 2 diabetes. Am J Clin Nutr. 2010;91(6):1777-1783.

26. Ahmed AT, Karter AJ, Warton EM, et al. The relationship between alcoho consumption and glycemic control among patients with diabetes:The Kaiser Permanente Northern California diabetes registry. $J$ Gen Intern Med. 2008;23(3):275-282.

27. Zhu W, Meng YF, Wu Y, et al. Association of alcohol intake with risk of diabetic retinopathy:a meta-analysis of observational studies. Sci Rep. 2017;7(1):4.
28. Dunkler D, Kohl M, Heinze G, et al. Modifiable lifestyle and social factors affect chronic kidney disease in high-risk individuals with type 2 diabetes mellitus. Kidney Int. 2015;87(4):784-791.

29. De Francisco ALM, Fresnedo GF, Palomar R, et al. The renal benefits of a healthy lifestyle. Kidney Int. 2005;68(Suppl 99):S2-S6.

30. Emanuele NV, Swade TF, Emanuele M. Consequences of alcohol use in diabetics. Alcohol Health Res World. 1998;22(3):211-219.

31. Koppes LL, Dekker JM, Hendriks HF, et al. Meta-analysis of the relationship between alcohol consumption and coronary heart disease and mortality in type 2 diabetic patients. Diabetologia. 2006;49(4):648-652.

32. Tanasescu M, Hu FB, Willett WC, et al. Alcohol consumption and risk of coronary heart disease among men with type 2 diabetes mellitus. $J \mathrm{Am}$ Coll Cardiol. 2001;38(7):1836-1842.

33. Valmadrid CT, Klein R, Moss SE, et al. Alcohol intake and the risk of coronary heart disease mortality in persons with older-onset diabetes mellitus. JAMA. 1999;282(3):239-246.

34. Diem P, Deplazes M, Fajfr R, et al. Effects of alcohol consumption on mortality in patients with Type 2 diabetes mellitus. Diabetologia. 2003;46(11):1581-1585.

35. Kunutsor SK, Apekey TA, Walley J. Liver aminotransferases and risk of incident type 2 diabetes:A systematic review and meta-analysis. Am J Epidemiol. 2013;178(2):159-171.

36. Chagas P, Mazocco L, Piccoli J da CE, et al. Association of alcohol consumption with coronary artery disease severity. Clin Nutr. 2016;36(4):1036-1039.

37. Shai I, Rimm EB, Schulze MB, et al. Moderate alcohol intake and markers of inflammation and endothelial dysfunction among diabetic men. Diabetologia. 2004;47(10):1760-1767. 